\title{
A Robust Compliant Grasper via Shape Deposition Manufacturing
}

\author{
Aaron M. Dollar, Student Member, IEEE, and Robert D. Howe, Member, IEEE
}

\begin{abstract}
Joint compliance can enable successful robot grasping despite uncertainties in target object location. Compliance also enhances manipulator robustness by minimizing contact forces in the event of unintended contacts or impacts. In this paper, we describe the design, fabrication, and evaluation of a novel compliant robotic grasper constructed using polymer-based shape deposition manufacturing. Joints are formed by elastomeric flexures, and actuator and sensor components are embedded in tough rigid polymers. The result is a robot gripper with the functionality of conventional metal prototypes for grasping in unstructured environments but with robustness properties that allow for large forces due to inadvertent contact.
\end{abstract}

Index Terms-Rapid prototyping, robotic grasping, robot hand design, shape deposition manufacturing, unstructured environments.

\section{INTRODUCTION}

$\mathrm{C}$ OMPLIANCE conveys two key advantages for robotic grasping: adaptability and robustness. In unstructured environments, sensing uncertainties are large and target object size and location may be poorly known. Finger compliance allows the gripper to conform to a wide range of objects while minimizing contact forces. Robot compliance or stiffness has often been considered in the context of active control, where sensors and actuators are used to achieve a desired force-deflection relationship [1]-[3]. In contrast, passive compliance, implemented through springs in robot joints, offers additional benefits, particularly in impacts, where control loop delays may lead to poor control of contact forces [4]-[8]. The elimination of the sensing required to create active compliance can also lower implementation costs.

Low joint stiffness can also enhance the robustness of robot grippers. Unintended contact can result in large contact forces unless the gripper is compliant. This contact can occur due to sensing uncertainty in unstructured environments, but can also happen in laboratory experiments, particularly in the debugging phase. Researchers are often reluctant to risk crashes with expensive multi-degree-of-freedom robot hands, so implementations must be carefully validated and experimental scope must be limited.

Designing durable robots, although rarely addressed in robotics research, is essential in industrial, space, and military applications. Examples include iRobot's "PackBot" [9], the Uni-

Manuscript received September 15, 2005; revised November 30, 2005. Recommended by Guest Editors S. Agrawal and V. Krovi. This work was supported in part by the Office of Naval Research Grant N00014-98-1-0669.

The authors are with the Division of Engineering and Applied Sciences, Harvard University, Cambridge, MA 02138 USA (e-mail: adollar@deas.harvard. edu; howe@deas.harvard.edu).

Digital Object Identifier 10.1109/TMECH.2006.871090 versity of Minnesota's "Scout” family of launchable robots [10], and MIT manipulator arms for the NASA/JPL Pathfinder and Surveyor Mars missions [11]. In research, this durability would expand the type of experimental tasks that can be reasonably attempted and speed implementation due to the reduced need for careful validation of programs.

In a previous work, we examined the optimization of the design of simple four-joint two-fingered grippers with passive springs in the joints [12]. This study showed that for a particular set of joint stiffnesses and rest angles, objects could be securely grasped for the widest range of uncertainty in object size and location. Contact forces were also minimized at approximately the same gripper configuration. In addition to simulation studies, these results were confirmed with experimental tests using a reconfigurable gripper with metal links and joint springs.

In this paper, we explore the benefits of using shape deposition manufacturing (SDM) for constructing this type of two-fingered gripper for unstructured environments [13], [14]. This process uses polymeric materials to simultaneously create the rigid links and compliant joints of the gripper, with embedded sensing and actuation components. In addition to simplifying the construction process, the result is an extremely robust gripper. Elastomeric polymers provide joint compliance, eliminating metal bearings, and tough rigid polymers fully encase the embedded components, eliminating the need for seams and fasteners that are often the source of mechanical failure. Our prototype is fully functional after impacts and other large loads due to unintended contact.

We begin the paper with a description of the SDM process and the design and manufacture of the prototype gripper. We then present experimental evaluation of the gripper, including informal evaluation of the gripper's robustness and a quantitative study of its performance in grasping, in comparison to both simulation results and the metal prototype's performance.

\section{DESIGN}

\section{A. SDM}

To fabricate our experimental grasper, we used SDM, an emergent manufacturing technique [13], [14]. This rapid prototyping process involves a cycle of deposition of part material and shaping, building up the part in distinct layers, and resulting in the concurrent manufacture and assembly of the part. In this way, the part can be manufactured in multiple sections or layers, allowing manipulation of the internal parts of the final structure. A diagram detailing the process is shown in Fig. 1 and an example use of the process with detailed steps is laid out in the next section. 


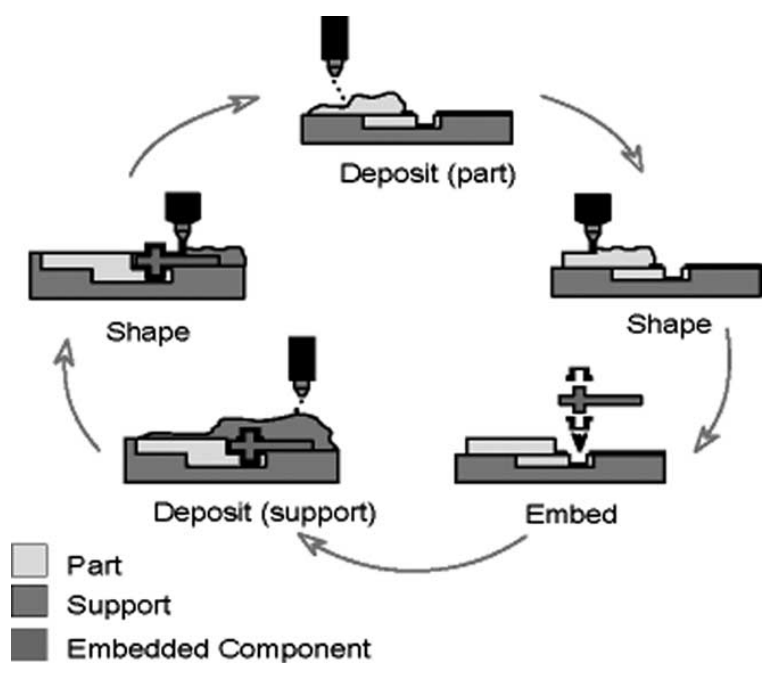

Fig. 1. The SDM process. Courtesy of Mark Cutkosky.

This process has a number of advantages over other prototyping techniques. The deposition of part material allows components to be embedded into the part during production, eliminating the need for fasteners and reducing the likelihood of damage to the component by encasing it within the part structure. This is a particularly desirable property for the inclusion of fragile components such as sensors, greatly increasing the robustness of the part. Also, depositing the part in layers permits the use of dissimilar materials, allowing for variation of mechanical properties within the same part. This property can be utilized to create complex mechanisms from a single part [15]-[17].

Due to its relative simplicity, custom tooling is not required to realize the SDM process. Complex part geometries can be attained using common computer numerical controlled (CNC) mill machines.

\section{B. Grasper Design and Fabrication}

Fig. 2 shows the steps of the SDM process used to produce our compliant grasper fingers. Pockets corresponding to the shape of the stiff links of our fingers are machined into a high-grade machine wax (Freeman Manufacturing and Supply Company Akron, OH, USA). The components in panel A are put into place in the pockets (panel B) and the polymer resin poured. Modeling clay is used to dam any areas to be blocked from the resin. After the layer cures, a second group of pockets is machined (both into the support wax and the stiff resin) and dammed (panel C). The polymer resins for the compliant finger joints (white) and soft fingerpads (clear) are then poured (panel D) and allowed to cure. The block is then faced off to level the surface and remove surface flaws (panel E), and the completed fingers removed from the wax support material. The entire process takes approximately $30 \mathrm{~h}$ to complete, only 4 of which require human intervention.

The polymers used are two-part industrial polyurethanes. Different compositions are used for the soft fingerpads, compliant joints, and stiff links (IE35A, IE90A, and IE72DC, respectively, Innovative Polymers, St. Johns, MI). Degassing at $-737 \mathrm{mmHg}$ $\left(-29^{\prime \prime} \mathrm{Hg}\right)$ was sometimes necessary to prevent voids in the cured resins. Table I shows material properties of these three polyurethanes as provided by the manufacturer.

Fig. 3 shows the parts of the SDM finger. The concave side of each link contains a soft fingerpad to maximize friction and increase grasp stability [18], [19]. The thin sections between links are the compliant joint flexures, designed to be compliant in the plane of finger motion and stiff out of plane. The joints are designed to have stiffnesses of 0.0421 and $0.224 \mathrm{~N} . \mathrm{m} / \mathrm{rad}$ for the proximal and distal joints, respectively, resulting in a proximal/distal stiffness ratio of 0.19 .

Conveniently, the polymer used for the stiff links is transparent, allowing the embedded components to be clearly seen (also see Fig 2(A)). Joint angle sensing is accomplished by embedding a low-output impedance linear hall-effect sensor (A3517SUA, Allegro MicroSystems, Inc., Worcester, MA) on one side of the joint and a rare-earth magnet $(6.35 \mathrm{~mm}$ diam $\times 3.18 \mathrm{~mm}, \mathrm{Nd}$ FeB, 10,800 Gauss strength, K\&D Magnetics, Inc., Boca Raton, FL) on the other side. Joint motion changes the distance between the two, varying the sensor output. The sensors are wired to exposed connectors (2.5-mm PC board header) for connection to external cables. A dovetail protrusion on the base link allows the finger to be securely connected to the grasper base.

For actuation, each finger has a prestretched nylon-coated stainless steel cable $(7 \times 7$ strand core, $0.94 \mathrm{~mm}$ diameter, $540 \mathrm{~N}$ breaking strength) anchored into the distal link. This cable runs through the bodies of the proximal and base links through lowfriction nylon 11 tubing ( $3.2 \mathrm{~mm}$ OD, $2 \mathrm{~mm} \mathrm{ID}$ ). Because of the joint compliance, the finger can be underactuated, allowing for one tendon cable to drive both joints. The grasper is intended to be unactuated until contact is made with the target object and a successful grasp is predicted based on the available sensory information. Before actuation, the tendon cable, which is in parallel with the compliant joints, remains slack and the finger is in its most compliant state. This method permits the use of actuators that are not backdrivable and prevents the inertial load of the actuator from increasing the passive stiffness. After actuation, the stiff tendon takes much of the compliance out of the fingers, resulting in a grasp with greater stability.

Fig. 4 shows the fully assembled grasper (two fingers, two motors, and base). The base was also produced using SDM, but is purely structural. The link lengths, measured from the centers of the joint flexures, were chosen to be equal to enable the tip to reach the base. The joint rest angles of the fingers $\left(25^{\circ}\right.$ and $45^{\circ}$ for the proximal and distal joints, respectively) were carefully chosen based on the results of previous optimization studies [12]. The ratio of joint stiffnesses ( 0.19 proximal/distal) was based on the optimization studies and additional material and geometric considerations to create a functional grasper. These angles and stiffnesses were shown to enable grasping of the widest range of object sizes with the greatest amount of uncertainty in object position.

The design is almost completely 2.5 dimensional (i.e., extruded two-dimensional shapes) and symmetric about the center plane, allowing for the same finger to be used on the right or left side of the grasper.

For comparison to the single-part SDM finger, a similar grasper made from aluminum that was used in previous work 

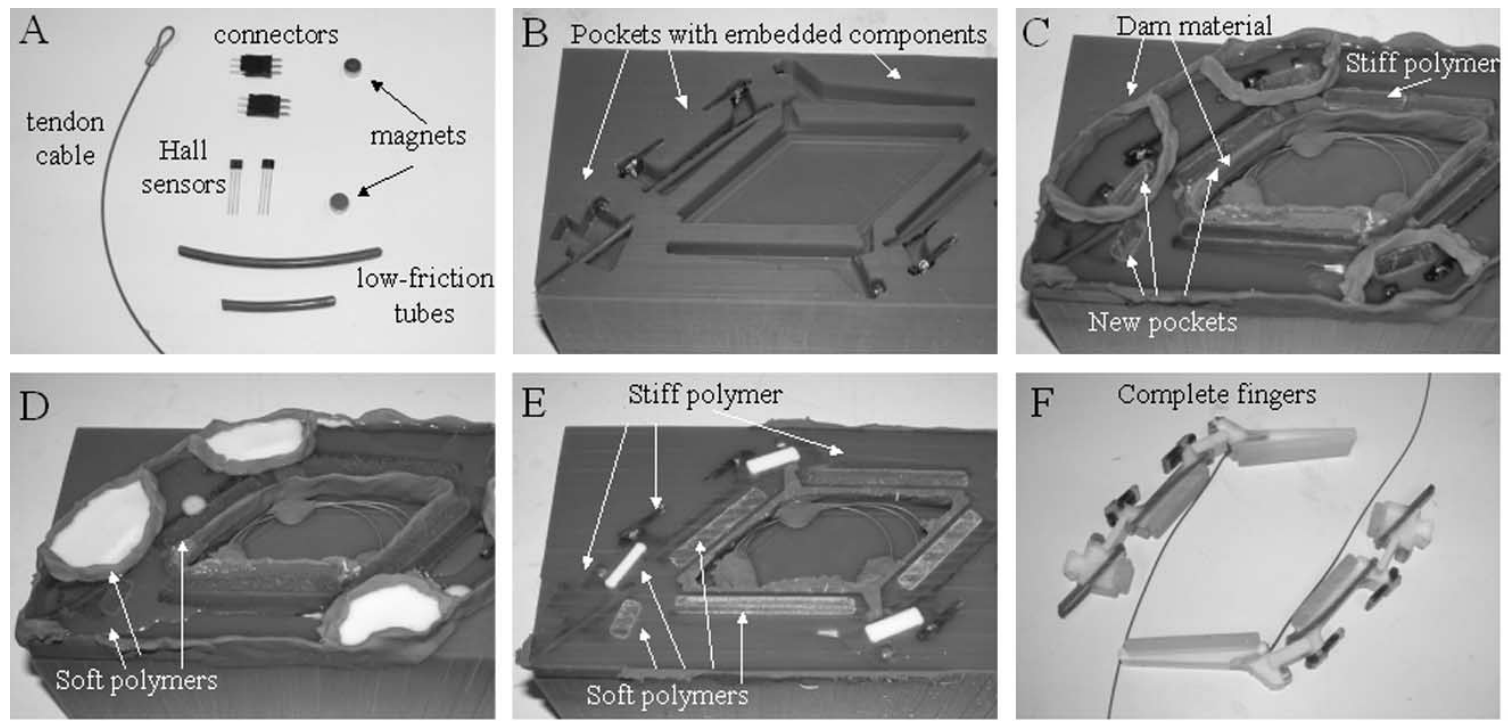

Fig. 2. Steps of the SDM process used to fabricate the grasper fingers.

TABLE I

MATERIALS SPECIFICATIONS

\begin{tabular}{lrrrr}
\hline & IE20AH & IE35A & IE90A & IE72DC \\
\hline \multicolumn{1}{c}{ Hardness } & $15-25$ & $30-40 \mathrm{~A}$ & $85-95 \mathrm{~A}$ & $75-85 \mathrm{D}$ \\
Tensile strength & & & & \\
ASTM D-638 (ksi) & 0.2 & 0.4 & 1.8 & 10 \\
$\begin{array}{l}\text { Elongation at break } \\
\text { Tear strength ASTM }\end{array}$ & $175 \%$ & $470 \%$ & $100 \%$ & $2 \%$ \\
$\begin{array}{l}\text { D-624 (pil) } \\
\text { Flex modulus ASTM }\end{array}$ & 25 & 50 & 250 & N/A \\
$\begin{array}{l}\text { D-790 (ksi) } \\
\text { Ultimate flex }\end{array}$ & N/A & N/A & N/A & 325 \\
Strength D-790 (ksi) & N/A & N/A & N/A & 13 \\
\hline
\end{tabular}

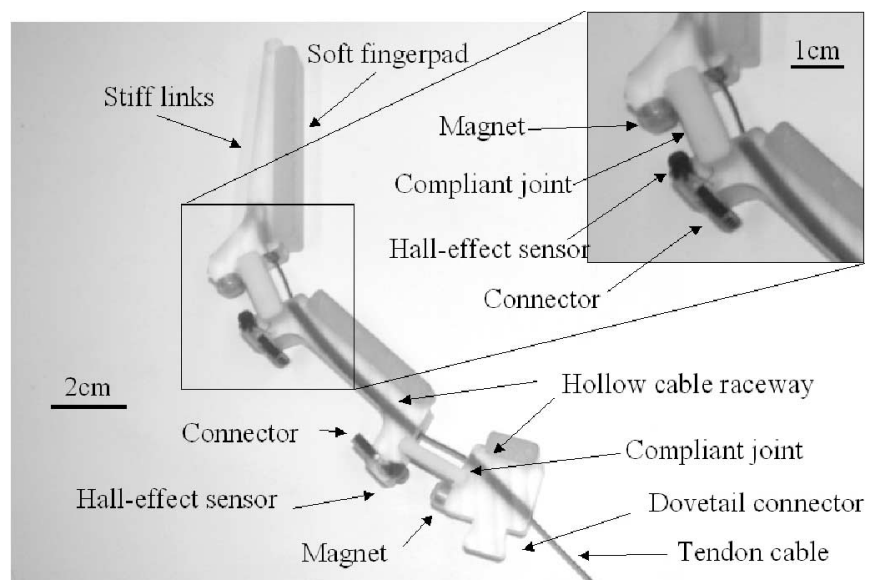

Fig. 3. Details of finger parts and placement of components.

is shown in Fig. 5 [12]. Each finger on this grasper contains over 60 distinct parts, 40 of which are fasteners! There is also a significant weight reduction in the SDM fingers (39 g each) versus the aluminum fingers of similar size ( $\sim 200 \mathrm{~g}$ each).

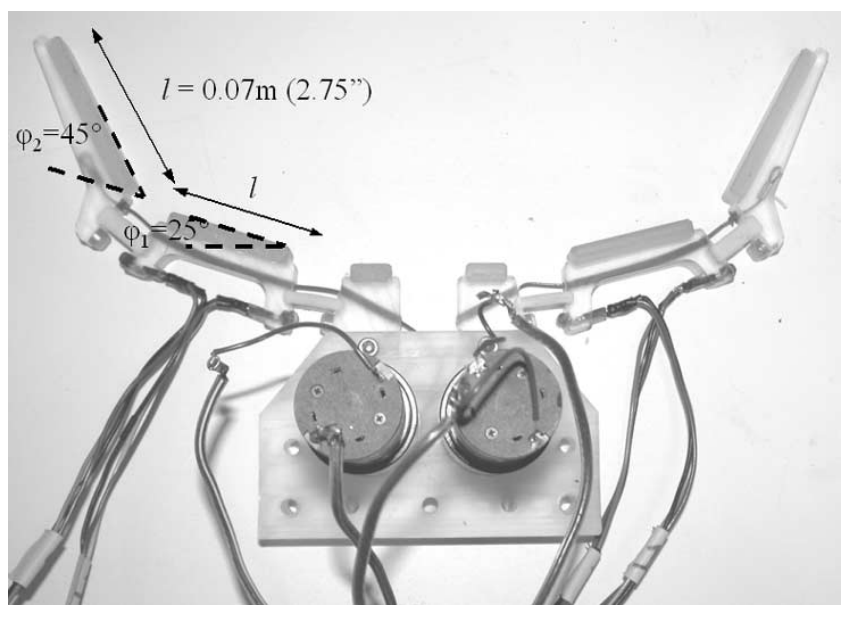

Fig. 4. Overhead view of the SDM grasper.

\section{Mechanism Behavior}

A number of tests were performed to characterize the behavior of the SDM grasper. The polyurethane used for the joints (IE90A) demonstrates significant viscoelastic behavior, as shown in Fig. 6. The sample tested corresponds to the dimensions of the distal joint flexure. A step angular displacement of $0.54 \mathrm{rad}$ was applied and the joint torsional stiffness was measured over a 30 -min interval.

The results show behavior consistent with a second-order Kelvin model [20], as shown in Fig. 6. Note the nonzero origin of the vertical axis, highlighting the second-order fit

$$
k_{\theta}=0.176+0.0303 e^{-0.0156 t}+0.0437 e^{-0.00125 t}
$$

where $k_{\theta}$ is in N.m/rad and $t$ is in seconds. Over the 30-min time interval tested, the joint torque drops $29 \%$. The time constants are much larger than typical grasp time, so the damping in the material has little effect on control of the grasper. 


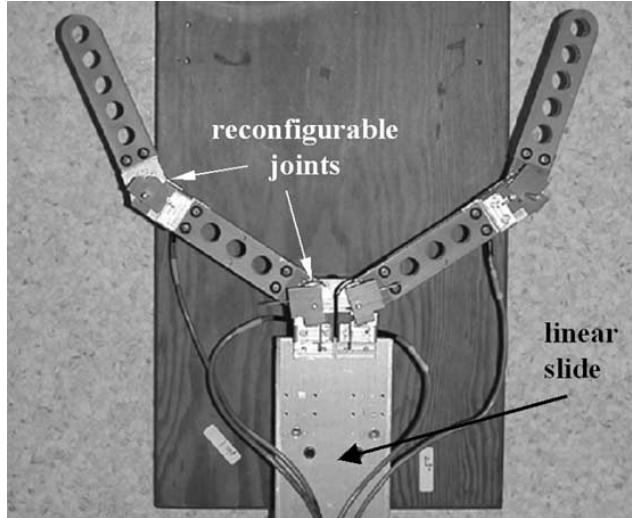

Fig. 5. Overhead view of the aluminum grasper.

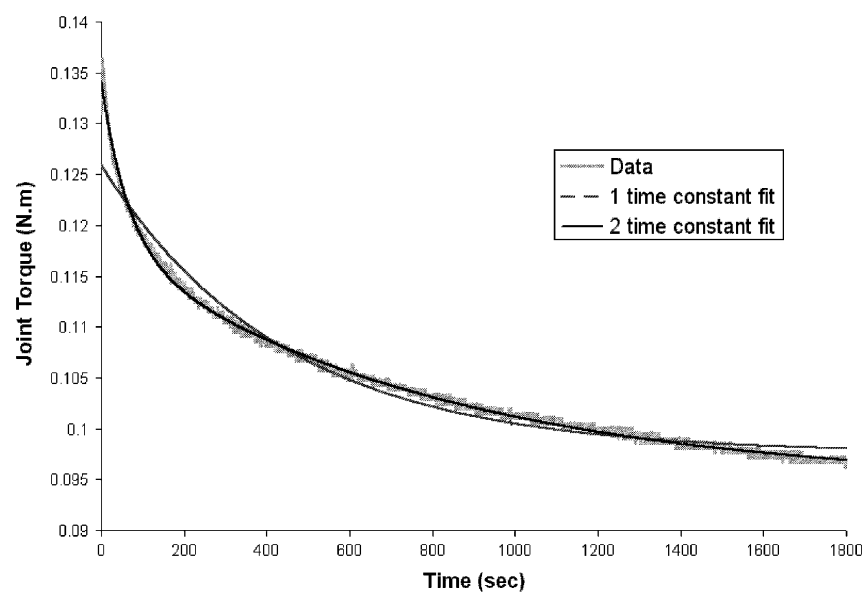

Fig. 6. Force relaxation of the distal joint of the SDM finger, for an angular step displacement of $0.54 \mathrm{rad}$.

The viscoelastic properties of the joint material have the beneficial effect of damping out joint oscillations caused by grasper accelerations. In an undamped compliant grasper, these oscillations can be large due to the significant moment of inertia about the joints caused by long finger links. This effect was observed in our previous prototype that used music wire torsional springs in the joints (Fig. 5) [12]. In this conventionally assembled grasper, oscillations due to large step displacements persisted for tens of seconds after release.

Low joint stiffness, although minimizing unwanted contact forces, increases the magnitude of resonant oscillations. Damping in the joints reduces the severity of these oscillations and therefore permits use of low joint stiffness. Fig. 7 shows the joint response of the SDM finger to a large step displacement of the fingertip, released at time $t=0$. Note that the oscillations are negligible after less than $1 \mathrm{~s}$.

Fig. 8 shows the torque and angular deflection behavior of the joints of the grasper for different joint flexure sizes. Loads were applied and removed quickly in order to minimize the effects of the material viscosity. Note that the joint angular deflections are nearly linearly proportional to load torque even across large deflections, allowing for the assumption of simple cantileveredbeam bending behavior.

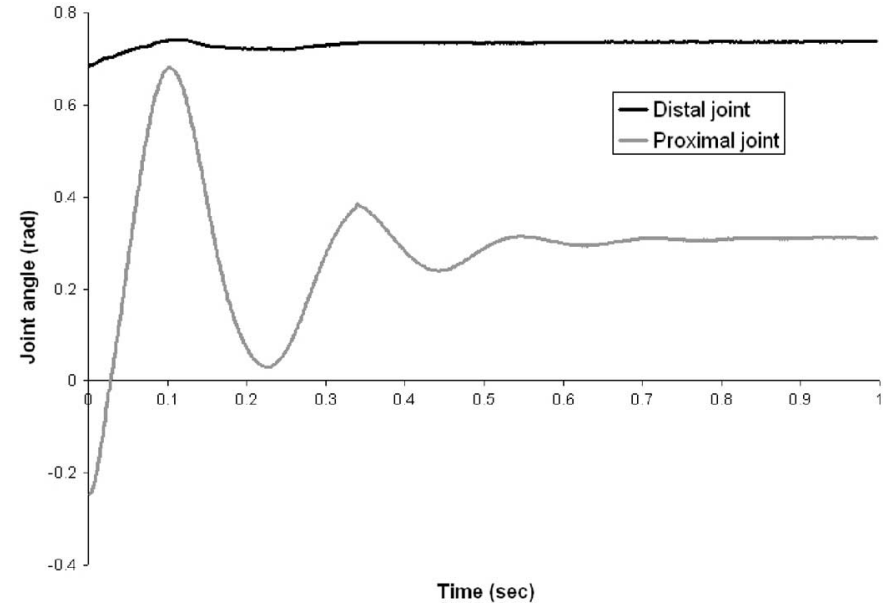

Fig. 7. Joint response of the SDM finger to a tip step displacement released at $t=0$.

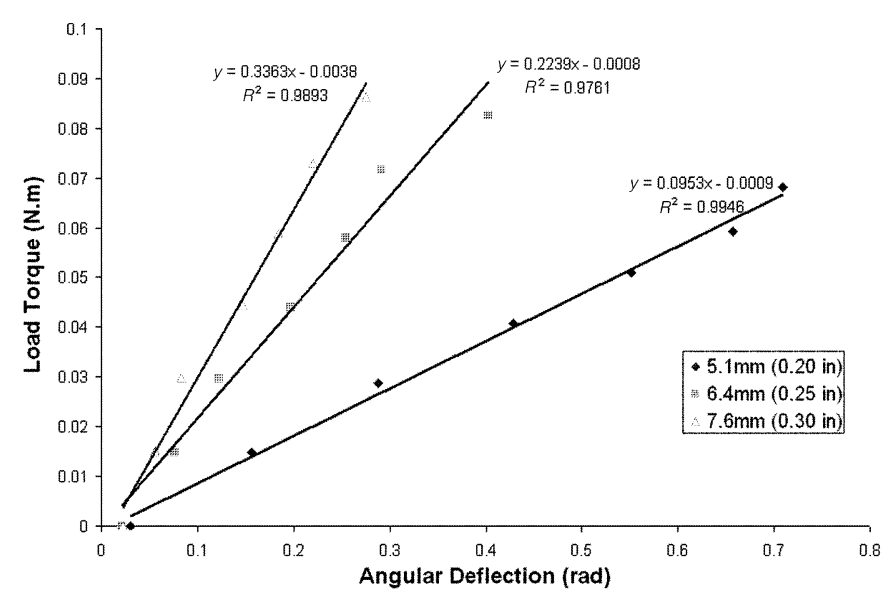

Fig. 8. Angular deflection of SDM joints as torque load is varied. Samples tested are 15.2-mm (0.6-in) long, 12.7-mm (0.5-in) deep, and varied thickness in the direction of load application.

Fig. 9 shows the behavior of the finger joints through their range of motion. Note that the center of rotation varies slightly with joint angle. Fig. 10 shows the output ' $V$ ' of the joint angle sensors (after amplification) and their fits versus joint deflection $\theta$ for the two fingers used in this study. The fit curves are of the form

$$
\theta=\left(c_{4} V^{4}+c_{3} V^{3}+c_{2} V^{2}+c_{1} V+c_{0}\right)^{-1}-1
$$

where $c_{i}$ are the fit coefficients. These sensors give sufficient sensitivity across the entire range of motion of the joints to allow for use in the control of the grasper.

Note that the sensor gives better resolution as the finger opens ( $\theta$ decreases) in order to optimize sensitivity during passive contact. This allows the grasper to be used as a "feeler."

Fig. 11 shows the joint deflection behavior as the finger is actuated without object contact. Note that the distal joint moves very little until the proximal joint completes its full range of motion, due to differences in joint stiffness and cable lever arm. This behavior is similar to that of the two distal joints of the human finger and increases the chances that both links of the 


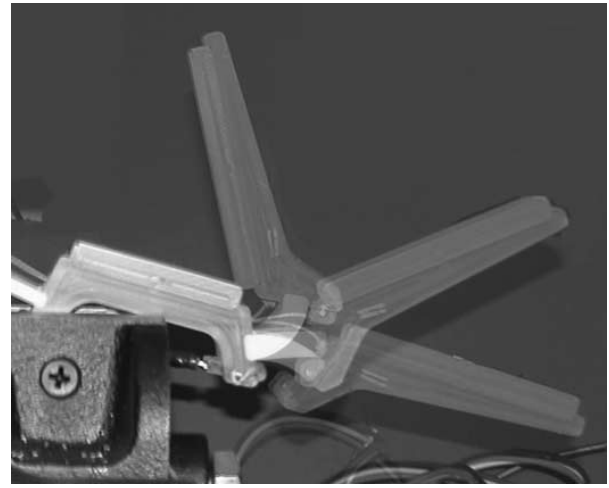

Fig. 9. Superimposed photograph of joint deflection and link motion for three positions across the travel range of the distal joint of the fingers. The center image is the rest position.

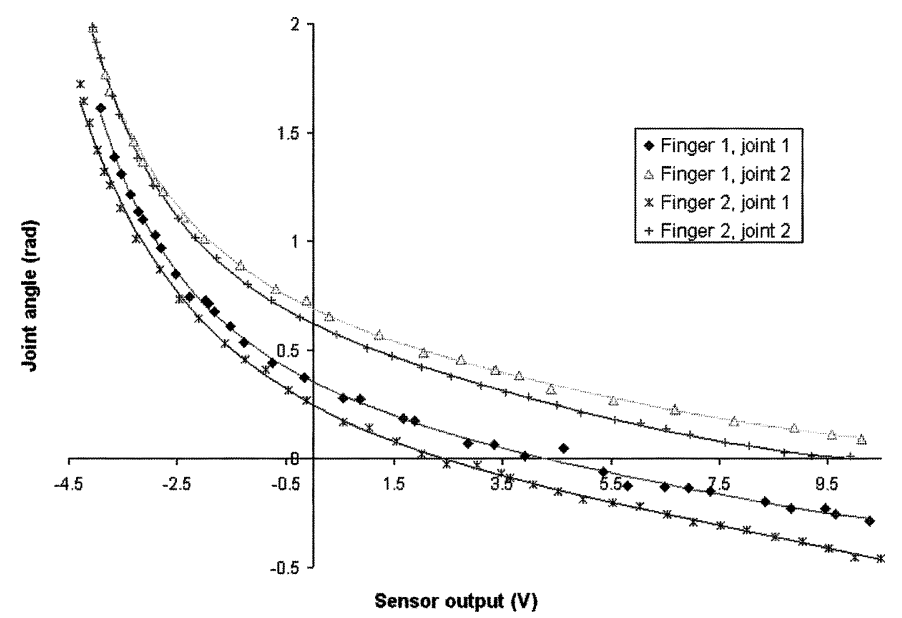

Fig. 10. Joint angle sensor calibration data and fits.

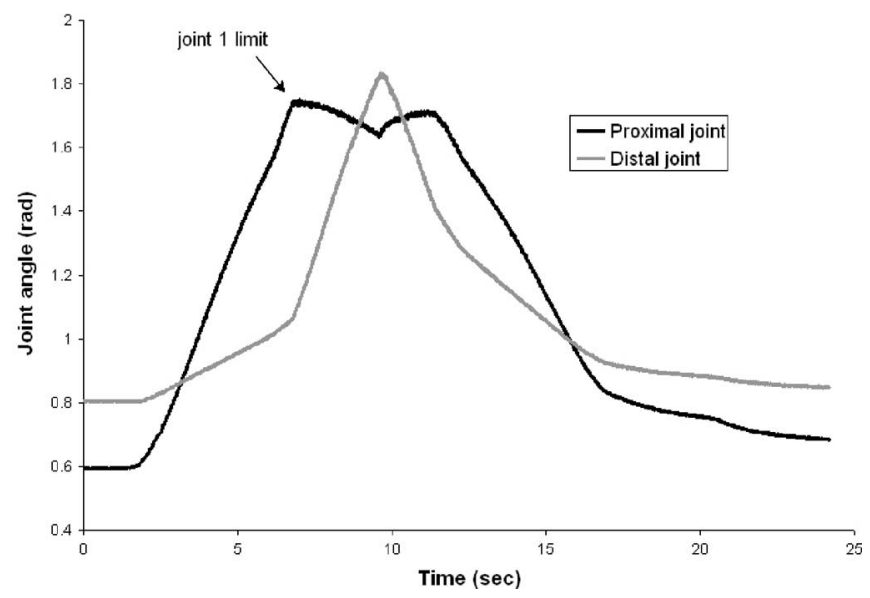

Fig. 11. Joint behavior as the finger is actuated without object contact.

finger are in contact with the object, increasing contact area and friction. The "dip" in the proximal joint curve is caused by outof-plane motion that occurs when a joint has reached its travel limit. The hall-effect sensors are only calibrated for motion in the plane.

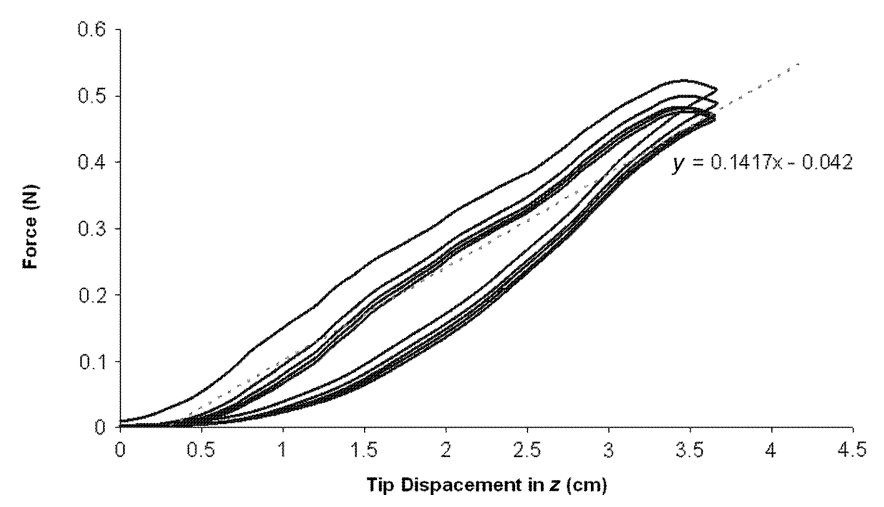

Fig. 12. Force-deflection curve of the tip of the SDM finger with linear trendline. The data represents five cycles of tip motion.

Fig. 12 shows the force generated at the tip of the fingers due to displacement in the out-of-plane direction ( $z$-direction following the convention of Fig. 16). The tip was displaced at a rate of approximately $1 \mathrm{~cm} / \mathrm{s}$ while mounted on an actuated linear slide mechanism (R2D series rodless actuator, Industrial Devices Corporation, Petaluma, CA). Force was measured with a multiaxis force/torque sensor (Gamma model, ATI Industrial Automation, Apex, NC). This data represents force generated due to motion of the tip across the tested range and back for a total of five cycles, then low pass filtered with a cutoff frequency of $1 \mathrm{~Hz}$, to remove sensor noise. Note the hysteresis in the curves and the force relaxation due to viscoelasticity. The data is fitted with a trend line to give an indication of the tip stiffness. The same tests were performed in the $x$ and $y$ directions (following the convention of Fig. 16) and show similar behavior. The approximate tip stiffness in the $x, y$, and $z$ directions are 5.85, 7.72, and $14.2 \mathrm{~N} / \mathrm{m}$, respectively.

The SDM fingers, while exhibiting low tip stiffness, can also undergo large deflections while remaining completely functional. In the test shown in Fig. 12, the tip was displaced more than $3 \mathrm{~cm}$ in the out-of-plane direction without any degradation of mechanical properties. The advantages of this property are clear when considering the usual result of unplanned contact during use of traditional research robotic hands.

The grasper does not exhibit this amount of compliance during all phases of the grasping task, however. Although not quantitatively evaluated, the grasper becomes much stiffer after it is actuated by cable - a desirable characteristic allowing for more accurate manipulation of the grasped object.

To give a sense of the robustness of the mechanism to impact loads, a more informal test was performed. An SDM finger was repeatedly dropped from a height of over $15 \mathrm{~m}$ onto a stone floor. After two attempts, no noticeable damage had occurred. After three, a small piece broke off of the dovetail connector. After six attempts, the outer link developed a large crack and one of the magnets broke off, but the sensors and joints remained intact and functional.

\section{Tactile Sensor}

A tactile sensor has been developed for integration with the soft fingerpads (Fig. 13). The sensor uses a reflective object 


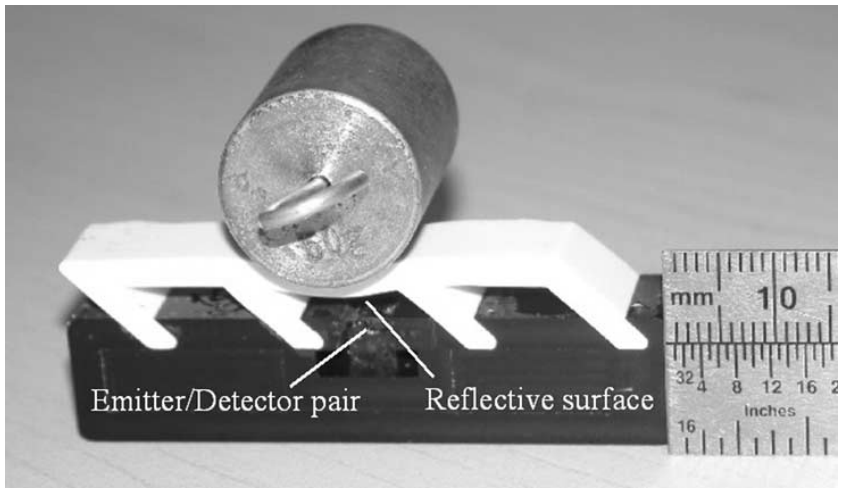

Fig. 13. Tactile sensor prototype with $50 \mathrm{~g}$ weight placed over the sensor. The angled strut flexures separate the reflective surface from the sensor face. Note the curvature of the reflective surface due to the applied load.

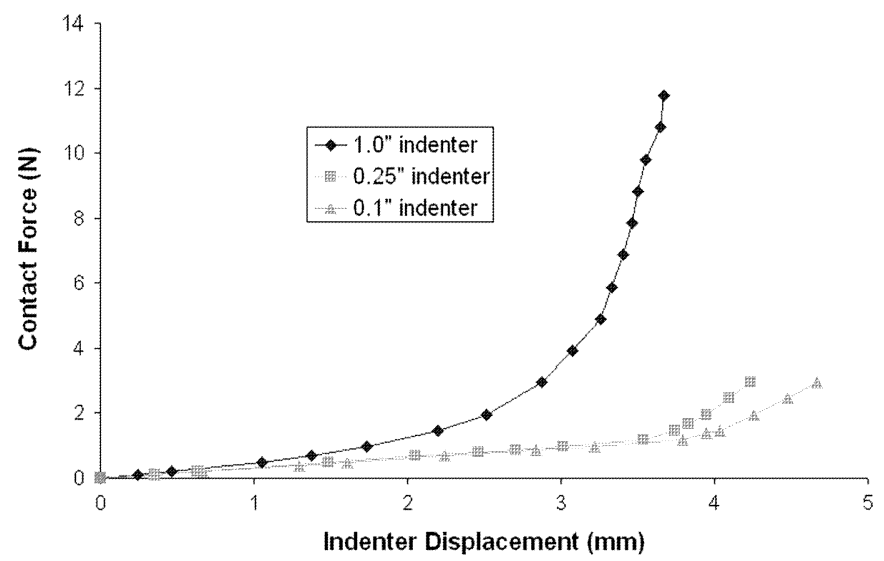

Fig. 14. Stiffness of the tactile sensor pad for various spherical indenter diameters.

sensor (OPB608R, 660-nm emitter wavelength, Optek Technology, Carrolton, TX) which consists of an LED and photodetector. As the finger applies force to an object, the pad deforms inwards, bringing the reflective inner surface of the fingerpad closer to the embedded sensor and causing a change in detector current. The slanted struts reduce stiffness in the normal contact direction. The sides of the sensor are easily enclosed to block ambient light from reaching the detector. Although this prototype contains only one optical sensor, multiple sensors can be embedded in the pad at about one every $15 \mathrm{~mm}$ under the current design.

As shown in Fig. 13, black dye was used in the support material (IE72DC, see Table I for material properties) to shield the sensor from ambient visible light coming through the otherwise clear material. White dye was used in the fingerpad material (IE20AH) to increase the reflecance and block ambient light from the front of the sensor.

Fig. 14 shows the effective stiffness of the fingerpad when loaded directly above the sensor. Loads were applied with three diameters of spherical indenter: $2.5,6.4$, and $25 \mathrm{~mm}$. The stiffness of the pad is low, on the order of $1 \mathrm{kN} / \mathrm{m}$, depending on contact location and geometry. Fig. 15 shows the sensor output as a function of applied force for the various indenter diame-

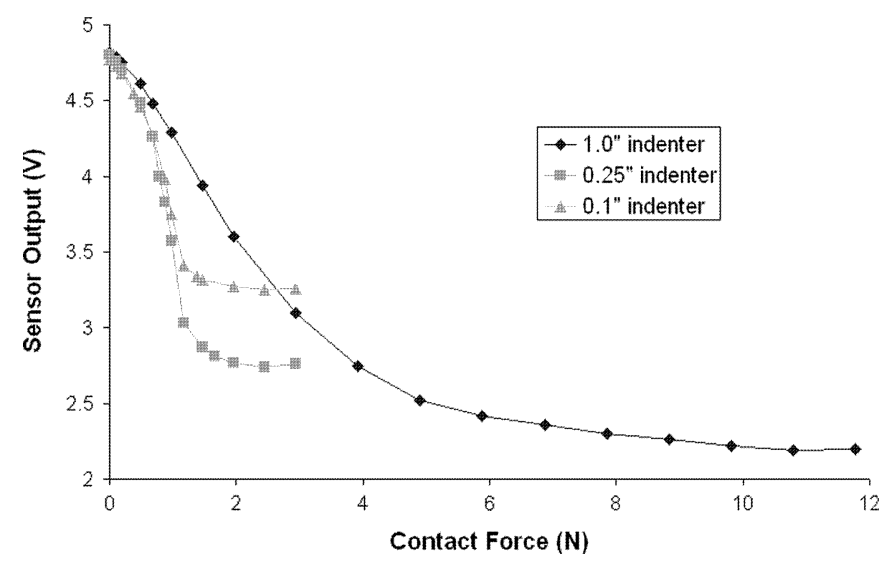

Fig. 15. Optical sensor output versus contact force for various spherical indenter geometries.

ters. It is clear from the figure that contact geometry plays a role in sensor output. This effect is due to both the difference in effective stiffness (as shown in Fig. 14) and the curvature of the reflective surface, which can deform with small objects to deflect light away from the detector. Note the higher sensitivity to smaller loads, a property useful in contact detection.

The sensitivity to contact geometry and limited force range reduces the usefulness of this sensor for contact force determination. However, the sensor provides an inexpensive method of sensing contact location with good sensitivity. This information can be useful in controlling contact force without force sensing as well as in determining object geometry based on contact location.

\section{EXPERIMENTAL EVALUATION}

\section{A. Experimental Apparatus and Procedure}

In an environment where sensing uncertainties are large, mechanical compliance can allow a robotic gripper to passively conform to the shape of the target object while minimizing contact forces. To maximize the effectiveness of the gripper, it should be designed to accommodate the largest range of target object size and location uncertainty. We evaluated the effectiveness of our compliant gripper by measuring the positions for which a successful grasp could be obtained for various object sizes. To accomplish this, the grasper was mounted on a precision screw-driven linear positioner, which brought the grasper into contact with the target object. The objects were positioned at increasing distances $x_{c}$ from the center of the grasper in the lateral $x$ direction, and securely mounted to prevent motion due to gripper-object contact forces. The experimental apparatus is shown in Fig. 16. The objects were metal cylinders chosen to reflect the sizes used in previous studies [12], and were mounted on a multiaxis force/torque sensor (Gamma model, ATI Industrial Automation, Apex, NC) to record the contact forces in the plane. Force was recorded at a resolution of $0.016 \mathrm{~N}$.

Joint angles and contact forces were recorded as the grasper moved forward along the linear actuator at a rate of $2 \mathrm{~cm} / \mathrm{s}$. Based on the joint angle information and knowledge of the object size and distance from the line of travel, the amount of object 


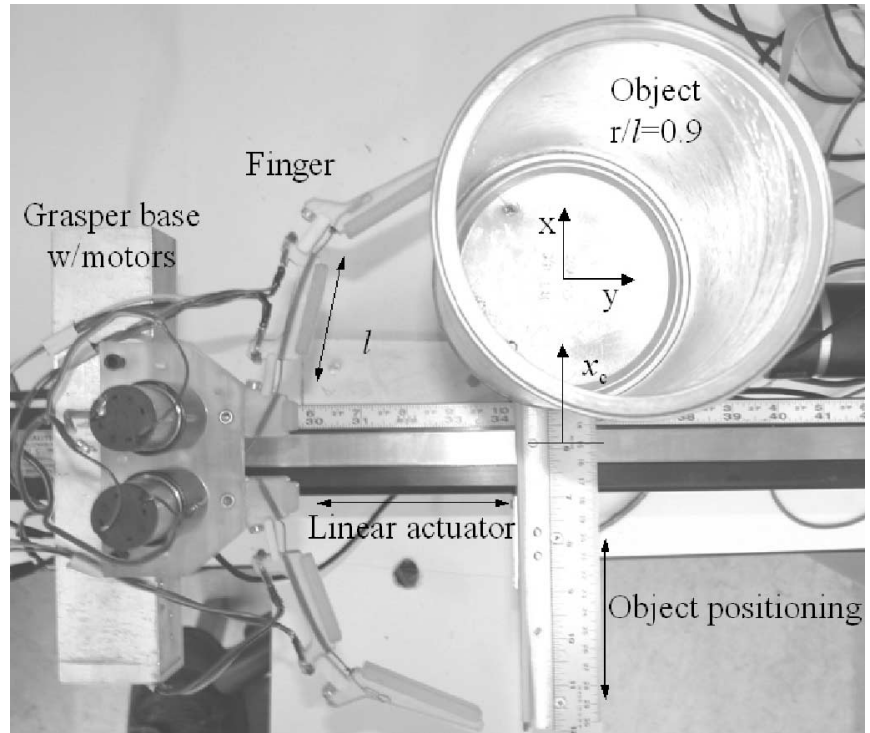

Fig. 16. Experimental setup. The grasper is mounted on an actuated linear slider and the object, affixed to a six-axis force/torque sensor, can be positioned at distances normal to the actuation direction.

TABLE II

NOMENCLATURE

\begin{tabular}{cl}
\hline Parameter & \multicolumn{1}{c}{ Definition } \\
\hline$\phi_{1}, \phi_{2}$ & spring rest link angles \\
$\theta_{1}, \theta_{2}$ & angular deflections from $\phi_{1}$ and $\phi_{2}$ \\
$k_{1}, k_{2}$ & joint stiffness values \\
$k_{\mathrm{T}}$ & total stiffness $\left(k_{1}, k_{2} / k_{1}+k_{2}\right)$ \\
$x_{\mathrm{c}}$ & distance from object center to the grasper centerline \\
$r$ & object radius \\
$l$ & grasper link length \\
$f_{R}$ & resultant contact force $=\sqrt{f_{T}^{2}+f_{N}^{2}}$ \\
$f_{T}$ & contact force tangential to the link surface \\
\hline
\end{tabular}

enclosure was calculated using the kinematics of the grasper and geometry of the object. If the grasper finger contacts can enclose greater than $180^{\circ}$ of the object surface, an enveloping grasp will be attained, and the grasp is deemed successful. For this evaluation of grasp range, the grasper is not actuated, but is allowed to passively conform to the shape of the target object. The kinematics of the grasper and object pair determines grasp success. See [12] for further discussion of this grasping scenario and success metric.

The performance of the grasper mechanism was evaluated for normalized object radius $r / l=0.5$ and 0.9 , and object location $x_{c} / l$ incremented by 0.023 from the center toward the outside of the grasping range, where $l$ represents the grasper link length (Table II). The maximum normalized distance of the object from the centerline for which a successful grasp was attained was recorded for each configuration. This value represents the successful grasp range and indicates the grasper's robustness to uncertainty in object location. The contact forces applied to the

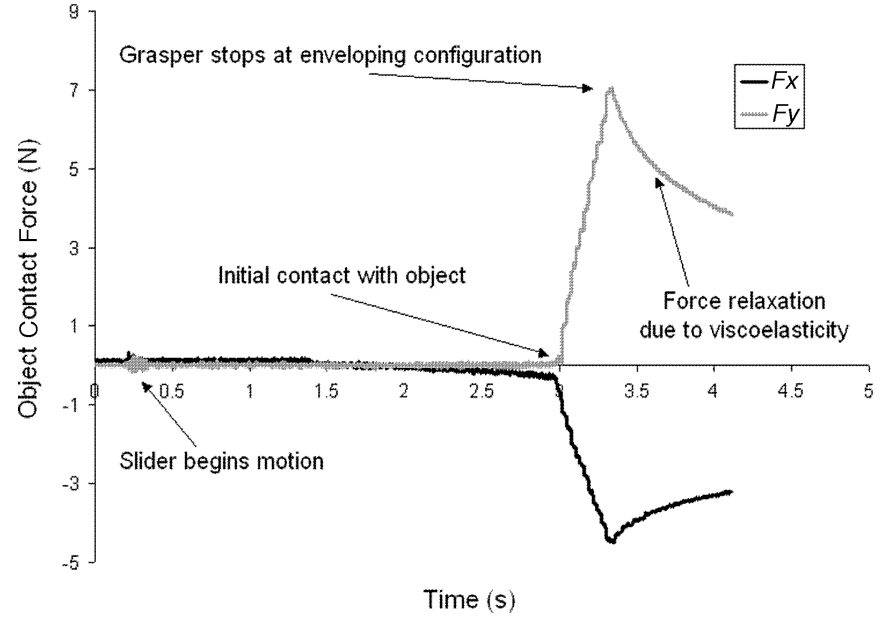

Fig. 17. Object forces due to grasper contact. The grasper moves forward at a constant velocity of $2 \mathrm{~cm} / \mathrm{s}$ until a successful grasp configuration is reached.

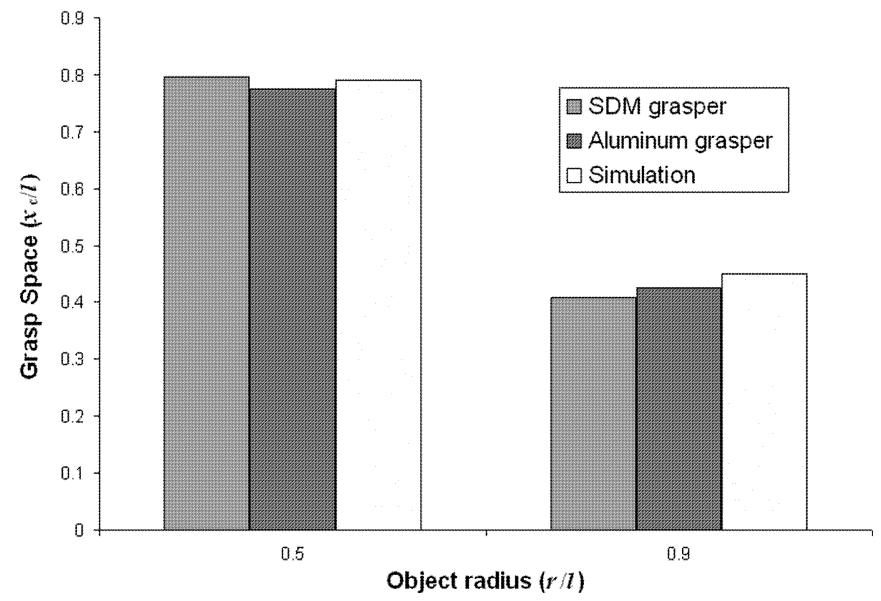

Fig. 18. Successful grasp range of the SDM grasper compared to the aluminum grasper and simulation.

object during the grasping process were also recorded for each tested value of object location $x_{c} / l$.

\section{B. Results}

Fig. 17 shows an example plot $\left(r / l=0.9\right.$ and $\left.x_{c} / l=0.45\right)$ of contact forces as the grasper moves forward against the object until a successful grasp configuration is obtained. As contact is made, the force causes deflection of the grasper, occurring primarily at the proximal joint, which is more compliant and is affected by a larger lever arm than the distal joint. This deflection continues as the grasper moves forward, with object force increasing nearly linearly, until an enveloping configuration has been reached. Force on the object due to the passive contact then decreases due to the viscoelasticity in the joint flexures and fingerpads.

Fig. 18 shows the successful grasp range of the SDM grasper and the analogous results from the aluminium grasper (Fig. 5) and simulation [12] for objects of radius $r / l=0.5$ and 0.9 . The object can be successfully grasped anywhere within this range, indicating the allowable uncertainty in object position for a 
successful grasp. The results show that the center of an object of radius $r / l=0.5$ can be located anywhere within the range $x_{c} / l= \pm 0.80$ from the centerline of the grasper. Similarly, a large object $(r / l=0.9)$ can be located anywhere within the range $x_{c} / l= \pm 0.41$. The values of the SDM grasp range show good agreement with the aluminum and simulated graspers.

\section{CONCLUSION AND FUTURE WORK}

Passive compliance confers a number of advantages for robotic hands. Previous studies showed that carefully tuned joint compliance maximizes the range of object positions that result in a successful grasp and minimizes the magnitude of forces that the grasper applies to the object [12]. These benefits are particularly important in unstructured environments, where object location and size may be poorly known.

In this paper, we present a gripper fabricated using a simple prototyping technique that minimizes construction complexity and increases robustness, while preserving the advantages of passive joint compliance. Robustness is a limiting factor in experimental development of multifingered robot hands: the expense and fragility of these hands precludes casual experimentation, restricting the type of experimental tasks that can be reasonably attempted and slowing implementation due to the need for careful validation of programs. The grasper design presented here demonstrates that polymer-based SDM allows the construction of fingers with the functionality of conventional metal prototypes but far superior robustness properties.

This study described the design, fabrication, and evaluation of a compliant grasper that has properties desirable for grasping with inherent uncertainty: large successful grasp range, low passive contact forces due to mechanical compliance, and robust construction. However, the performance of the grasper has only been evaluated in structured tasks that foreshadow the performance in unstructured tasks. The natural extension of this work, therefore, is testing with more unstructured tasks, relaxing the assumptions of object geometry and position, and the requirement of an enveloping grasp for grasp success.

\section{ACKNOWLEDGMENT}

The authors would like to thank Mark Cutkosky, Moto Hatanaka, and Miguel Piedrahita for their advice and assistance with implementing the SDM process. Also, we would like to thank Chris Johnson for his assistance in implementing the process, and Francisco Isenberg for his work on the construction of the tactile sensor.

\section{REFERENCES}

[1] K. J. Salisbury, "Active stiffness control of a manipulator in Cartesian coordinates," in Proc. 19th IEEE Conf. Decision Control, 1980, pp. 95100 .

[2] M. R. Cutkosky and I. Kao, "Computing and controlling the compliance of a robotic hand," IEEE Trans. Robot. Autom., vol. 5, no. 2, pp. 151-165, Apr. 1989.

[3] J. P. Desai and R. D. Howe, "Towards the development of a humanoid arm by minimizing interaction forces through minimum impedance control," in Proc. 2001 IEEE Int. Conf. Robot. Autom., 2001, pp. 4214-4219.

[4] J. Loncaric "Geometrical analysis of compliant mechanisms in robotics," Ph.D. thesis, Harvard Univ., Cambridge, MA, 1985.
[5] D. E. Whitney, "Quasi-static assembly of compliantly supported rigid parts," J. Dyn. Syst. Meas. Control, vol. 104, pp. 65-77, 1982.

[6] J. M. Schimmels and S. Huang, "A passive mechanism that improves robotic positioning through compliance and constraint," Robot. Comput.Integr. Manuf., vol. 12, no. 1, pp. 65-71, 1996.

[7] L. Biagiotti, F. Lotti, C. Melchiorri, and G. Vassura, "Mechatronic design of innovative fingers for anthropomorphic robot hands," in Proc. 2003 IEEE Int. Conf. Robot. Autom., 2003, pp. 3187-3192.

[8] S. Hirose and Y. Umetani, "The development of soft gripper for the versatile robot hand," Mach. Machine Theory, vol. 13, pp. 351-359, 1978.

[9] iRobot CorporationGovernment and Industrial Robotics Division, (www.irobot.com/governmentindustrial), Burlington, MA, USA.

[10] D. F. Hougen et al., "A miniature robotic system for reconnaissance and surveillance," in Proc. IEEE Int. Conf. Robot. Autom., San Francisco, CA, Apr. 2000, pp. 501-507.

[11] D. A. Theobald et al., "Autonomous rock acquisition," presented at the AIAA Forum on Advanced Developments in Space Robotics, Madison, WI, Aug. 1-2, 1996.

[12] A. M. Dollar and R. D. Howe, "Towards grasping in unstructured environments: Grasper compliance and configuration optimization," Adv. Robot., vol. 19 , no. 5, pp. 523-544, 2005.

[13] R. Merz, F. B. Prinz, K. Ramaswami, M. Terk, and L. Weiss, "Shape deposition manufacturing," presented at the Solid Freeform Fabrication Symp., University of Texas, Austin, TX, Aug. 8-10, 1994.

[14] M. Binnard and M. R. Cutkosky, "A design by composition approach for layered manufacturing," ASME Trans., J. Mech. Design, vol. 122, no. 1, pp. 91-101, 2000.

[15] C. Stefanini, M. R. Cutkosky, and P. Dario, "A high force miniature gripper fabricated via shape deposition manufacturing," presented at the $2003 \mathrm{Int}$ Conf. Robot. Autom., Taipei, Taiwan, 2003.

[16] J. E. Clark, J. G. Cham, S. A. Bailey, E. M. Froehlich, P. K. Nahata, R. J. Full, and M. R. Cutkosky, "Biomimetic design and fabrication of a hexapedal running robot," presented at the Int. Conf. Robot. Autom. Seoul, South Korea, 2001.

[17] B. H. Park, M. Shantz, and F. B. Prinz, "Scalable rotary actuators with embedded shape memory alloys," Proc. SPIE., vol. 4327, pp. 79-87, 2001

[18] K. B. Shimoga and A. A. Goldenberg, "Soft materials for robotic fingers," in Proc. 1992 IEEE Int. Conf. Robotics and Automation, 1992, pp. 13001305.

[19] M. R. Cutkosky, J. M. Jourdain, and P. K. Wright, "Skin materials for robotic fingers," in Proc. IEEE Int. Conf. Robot. Autom., 1987, pp. 16491654.

[20] Y. C. Fung, Biomechanics: Mechanical Properties of Living Tissues. 2nd ed. New York: Springer-Verlag, 1993.

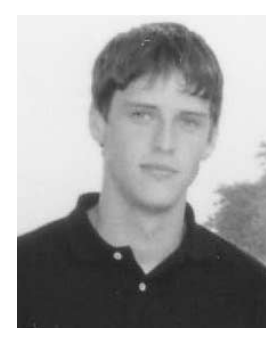

Aaron M. Dollar (S'02) received the B.S. degree in mechanical engineering from the University of Massachusetts, Amherst, in 2000, and the S.M. degree in engineering from Harvard University, Cambridge, MA, in 2002 . He is currently pursuing the $\mathrm{Ph}$.D. degree at the Division of Engineering and Applied Sciences, Harvard University.

His research focuses on mechanically compliant robot graspers and their application in unstructured environments. Mr. Dollar is a student member of the ASME and ASEE.

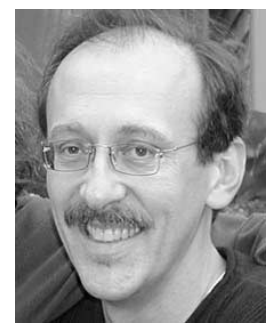

Robert D. Howe (S'88-M'99) received the B.A. degree in physics from Reed College, Portland, OR, and the Ph.D. degree in mechanical engineering from Stanford University, Stanford, CA, in 1990.

$\mathrm{He}$ has worked in the electronics industry as an analog and digital Design Engineer. Currently, he is the Gordon McKay Professor of Engineering the Division of Engineering and Applied Sciences, Harvard University, Cambridge, MA. His research interests focus on robot and human manipulation and the sense of touch. Bioengineering applications include the characterization of the mechanical properties of soft tissues and development of new techniques for image-guided surgery. 